\title{
Multi-loop PID Controller Design for Distillation Column using Firefly Algorithm
}

\author{
K. Saroja ${ }^{1}$, R. Stefka Sharon ${ }^{1}$, S. Meena ${ }^{2}$ and K. Chitra ${ }^{3}$ \\ Department of EIE, St. Joseph's College of Engineering, Chennai-600119, India ${ }^{1}$. \\ Research scholar, Faculty of Electronics engineering, Sathyabama University, Chennai-600119, India ${ }^{2}$. \\ Professor, SENSE, VIT University, Chennai, India ${ }^{3}$. \\ Sarojamoorthyk96@gmail.com¹,stefkasharon0596@gmail.com¹, meena27681@gmail.com², \\ kchitra68@gmail.com ${ }^{3}$
}

\begin{abstract}
The main motivation of this manuscript is to develop a PID tuning procedure to improve the performance of the benchmark distillation column models using the heuristic firefly algorithm. A decentralised PID tuning approach is developed to design an independent PID controller for the top and bottom product loop. Minimization of a multi-objective function is chosen to guide the search process of the controller tuning procedure. The performance of the proposed approach is confirmed by evaluating the time domain parameters and the error values. From the experimental results, the main observation is that, proposed approach offers satisfactory result on the proposed approach and helps to achieve a reduced values of the time domain and error values. The proposed approach proves its efficiency in a benchmark Two Input Two Output (TITO) system. In future, this approach can be adopted to design controller for the multi-loop and non-linear systems existing in the process control literature. The proposed approach can also be compared against other heuristic approaches existing in the literature.
\end{abstract}

Key words: PID controller, distillation column, firefly algorithm, performance evaluation.

\section{INTRODUCTON}

In the chemical industry, design of optimal controller is widely preferred to maximize the profit by minimizing the waste. Due to the economical and environmental point of aspect, safe working condition is always preferred in chemical process loops. Implementation of a suitable controller structure is one on the technique to maintain the working process loop in its safe region ${ }^{[1-4]}$. In the literature, a number of controlling procedures are proposed and implemented to operate the process loops in a safer working region. In real time cases, the widely preferred controllers are PI/PID, since these controller structures are simple to understand and easy to implement ${ }^{[5-12]}$.

In the proposed work, design of a suitable PID controller is discussed on a well known bench mark distillation process model. The distillation process is a really a complex unit with a series of actions or steps taken to achieve a particular end product with enhanced accuracy and purity. It is a series of interrelated tasks that, together, transform inputs into outputs ${ }^{[5-7]}$. Chemical, physical, electrical or mechanical procedures are the steps involved in industrial processes to aid in the manufacturing of an item, mostly carried out on a very large scale. Among the industrial process we use chemical process as they are multivariable in nature. Chemical process is a method intended to be used in manufacturing or on an industrial scale to change the composition of materials, usually using technology similar or related to that used in industry. Distillation column is said to be a separation process used for the purification of final products is widely implemented in chemical and petroleum industries. Due to their industrial significance they are generally used by the researchers.

Mostly industrial distillation processes are Multi-Input and Multi-Output (MIMO) in nature,i.e. a change or disturbance within a loop which causes to change the other loops through the cross-couplings between them, is the most important feature of a MIMO system. MIMO process is more complicated than a Single Input Single Output (SISO) process, but when implemented with a heuristic algorithm gives a better result with minimal errors $^{[13]}$.

In control process the literature gives us the modern and traditional approach for the design and optimization of PID controller. In this paper, PID controller design for MIMO process is addressed. The PID controller design is categorized as centralized and decentralized. Centralized system is far more complex than the decentralized 
system due to the interaction between the loops. The heuristic algorithm is assisted with the initial parameters which is the centralized system for a TITO process is proposed in this work.

The remaining sections of this paper are arranged as follows. Section 2 gives the process description of distillation column with its bench mark models. Section 3 describes methodology adopted in this work. Section 4 gives a detailed results of this work and the corresponding discussions over it. The conclusion of the present work is discussed in section 5 .

\section{PROCESS DESCRIPTION}

The distillation column is one of the important devices to support liquid-liquid separation processes in the chemical industry. It works under the application of the removal of heat to exploit difference in rapid change unpredictably. Distillation can be used to separate binary or multi-component mixtures. Many variables, such as column pressure, temperature, size, and diameter are determined by the properties of the feed and the desired products. Fig 1 depicts the basic distillation column existing in the literature ${ }^{[1,5-7]}$.

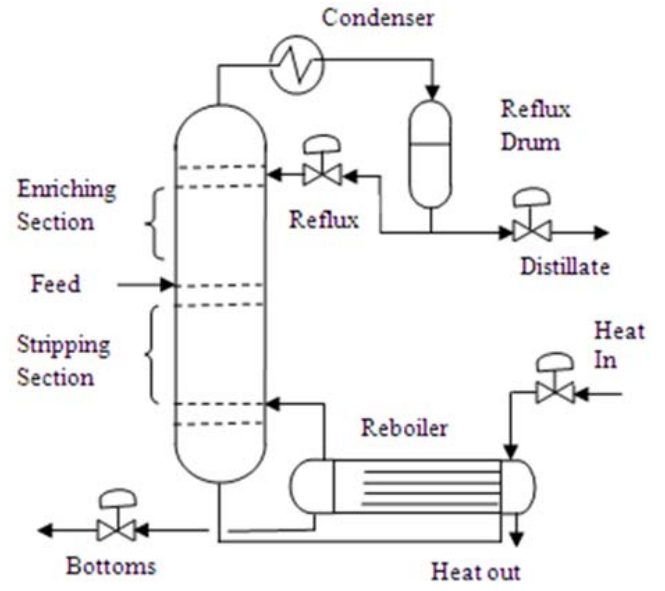

Fig. 1 Construction of distillation column

The distillation column is used to convert the raw materials into final products. The mixture is applied on the distillation column, with a number of trays and a number of temperature regions. Based on the column temperature, the mixture is split into the Liquid $(\mathrm{L})$ and the Vapour $(\mathrm{V})$ products. The process for the distillation column is based on L-V structure or the energy balance method. In this control configuration, the vapour flow rate and the liquid flow rate are the control inputs. In figure 1 the schematic of the distillation column is shown. The benchmark distillation columns are WW and $\mathrm{VL}^{[14-16]}$.

Eqn. 1 shows the mathematical model of VL system widely considered by the researchers;

$$
G(s)=\left[\begin{array}{cc}
-\frac{2.2}{7 s+1} e^{-s} & \frac{1.3}{7 s+1} e^{-0.3 s} \\
-\frac{2.8}{9.5 s+1} e^{-1.8 s} & \frac{4.3}{9.2 s+1} e^{-0.35 s}
\end{array}\right]
$$

Eqn. 2 shows the mathematical model of WW model;

$$
G(s)=\left[\begin{array}{lc}
\frac{0.126}{60 s+1} e^{-6 s} & \frac{-0.101}{(48 s+1)(45 s+1)} e^{-12 s} \\
\frac{0.094}{38 s+1} e^{-8 s} & \frac{-0.12}{35 s+1} e^{-8 s}
\end{array}\right]
$$

The major aim of this work is to design a suitable PID controller using recent heuristic algorithms. 


\section{METHODOLOGY}

In this work, design of a centralised PID is addressed using the well known Firefly algorithm and its performance is validated against the PSO and BFO algorithms.

a. Firefly algorithm

The classical Firefly Algorithm (FA) was initially proposed by Yang ${ }^{[17]}$. FA is a nature inspired meta-heuristic algorithm, in which flashing illumination patterns generated by fireflies are modeled using a suitable mathematical expression. In this work, the recent version of the FA discussed in ${ }^{[18-20]}$ is considered.

\section{START;}

Initialize essential algorithm parameters, $D$ and $f(T)$;

Generate initial locations of ' $n$ ' fireflies for $x_{i}(i=1,2, \ldots \mathrm{n})$

Determine the intensity of $i^{\text {th }}$ firefly based on $i^{\text {th }} f(T)$ value

If iter $<\mathrm{M}_{\mathrm{iter}}$;

For $i=1,2, \ldots, \mathrm{n}$;

For $j=1,2, \ldots, \mathrm{n}$;

If intensity of firefly $j>i$,

Calculate the Cartesian distance and move the $i$ towards $j$;

\section{End if;}

Repeat the above steps until iter $=$ Miter;

Estimate light intensity and update firefly positions;

End for $j$;

End for i;

Sort the fireflies in descending order based on the rank and find the optimal value;

End if:

Record the $f(T)$ and optimal threshold values.

STOP;

The pseudo code of FA is presented

The updated position of a firefly in a $D$ - dimensional search space can be expressed as;

$$
X_{i}^{t+1}=X_{i}^{t}+\beta_{0} \mathrm{e}^{-\gamma \mathrm{d}_{\mathrm{ij}}^{2}}\left(X_{j}^{t}-X_{i}^{t}\right)+\alpha_{1} \cdot \operatorname{sign}(\text { rand - } 1 / 2) \oplus A \cdot|s|^{\alpha / 2}
$$

where, $X_{i}^{t+1}$ is updated position of $i^{t h}$ firefly, $X_{i}^{t}$ is initial position of $i^{\text {th }}$ firefly, $\beta_{0} \mathrm{e}^{-\gamma \mathrm{d}_{\mathrm{ij}}^{2}}\left(X_{j}^{t}-X_{i}^{t}\right)$ is the attraction between fireflies, $\mathrm{A}$ is a random variable, $\beta$ is the spatial exponent, $\alpha$ is the temporal exponent, and $\Gamma(\beta)$ is the Gamma function. The details of FA can be found in the literature. The FA parameters are assigned as; dimension of the search $D=T$; population of firefly is chosen as 30 , number of iterations are assigned as 1000 and the stopping criteria is the $f(T)$.

b. Particle swarm optimization

PSO is a population based stochastic optimization technique inspired by social behaviour of bird flocking or fish schooling, and it is widely used in engineering applications due to its high computational efficiency. PSO algorithm is easy to implement and there are few parameters to adjust compared to other heuristic methods. It is a population based evolutionary computation technique, attempts to mimic the natural process of group communication of individual knowledge, to achieve some optimum property. It has two basic equations namely the velocity update and position update as presented below ${ }^{[21]}$ :

$$
\begin{aligned}
& V_{i}(t+1)=W^{t} . V_{i}^{t}+C_{1} R_{1}\left(P_{i}^{t}-S_{i}^{t}\right)+C_{2} R_{2}\left(G_{i}^{t}-S_{i}^{t}\right) \\
& X_{i}(t+1)=X_{i}^{t}+V_{i}(t+1)
\end{aligned}
$$

where $W^{t}=$ inertia weight coefficient (typically 0.7$), V_{i}^{t}=$ current velocity of particle, $V_{i}(t+1)=$ updated velocity of particle, $X_{i}^{t}=$ current position of particle, $X_{i}(t+1)=$ updated position of particle, $R_{l}$ and $R_{2}$ are 
random numbers between $\{0,1\}, \quad C_{1}=$ cognitive coefficient (typically 2.0), and $C_{2}=$ social coefficient (typically 1.8).

c. Bacterial foraging optimization

Bacteria Foraging Optimization (BFO) algorithm is a biologically inspired stochastic search technique based on mimicking the foraging (methods for locating, handling and ingesting food). During foraging, a bacterium can exhibit two different actions: Tumbling or swimming. The tumble action modifies the orientation of the bacterium. During swimming (chemotactic step) the bacterium will move in its current direction. Chemotactic movement is continued until a bacterium goes in the direction of positive nutrient gradient. After a certain number of complete swims, the best half of the population undergoes reproduction, eliminating the rest of the population. In order to escape local optima, an elimination-dispersion event is carried out where, some bacteria are liquidated at random with a very small probability and the new replacements are initialized at random locations of the search space.

In the proposed work, the enhanced BFO algorithm discussed in ${ }^{[22]}$ is adopted. The initial BFO parameters are assigned as follows:

Number of E.Coli bacteria $=N=30, N_{c}=\frac{N}{2} ; N_{s}=N_{r e} \approx \frac{N}{3} ; N_{e d} \approx \frac{N}{4} ; N_{r}=\frac{N}{2} ; \mathrm{P}_{\mathrm{ed}}=\left(\frac{N_{e d}}{N+N_{r}}\right)$;

$\mathrm{d}_{\text {attract }}=\mathrm{W}_{\text {attract }}=\frac{N_{s}}{N} ;$ and $\mathrm{h}_{\text {repell }}=\mathrm{W}_{\text {repell }}=\frac{N_{c}}{N}$

A detailed description of the considered BFO algorithm can be found in [10].

d. Implementation

This section discusses the implementation of the proposed controller design procedure for the chosen MIMO process models. Fig. 2 depicts the implementation of the proposed centralised PID design procedure.

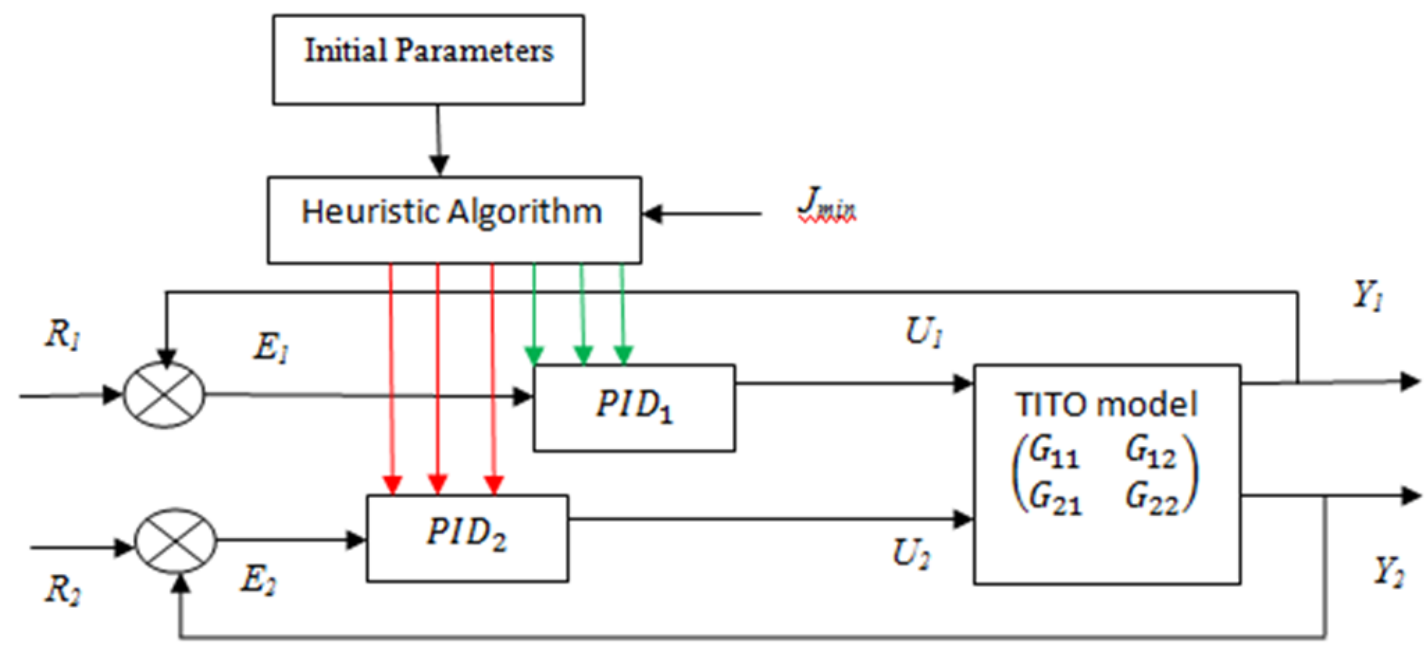

Fig. 2 Implementation of the proposed PID tuning approach

This process has two controllers, such as $\mathrm{PID}_{1}$ and $\mathrm{PID}_{2}$. Both the PIDs have the similar structure and the mathematical expression is given below ${ }^{23}$ :

$$
\begin{aligned}
& \mathrm{PID}_{1}=K_{p 1}+\frac{K_{i 1}}{s}+\frac{K_{d 1} s}{T_{f} s+1} \\
& \mathrm{PID}_{2}=K_{p 2}+\frac{K_{i 2}}{s}+\frac{K_{d 2} s}{T_{f} s+1}
\end{aligned}
$$


where $T_{f}=T_{d} / N_{f} ; T_{d}=$ derivative controller time constant; and $N_{f}=10$.

In this paper the heuristic algorithm, known as FA, which was first recently inspired by the nature of fireflies and the technique proposed is assisted by a PID controller design for the most common multi-variable system such as, Wardle-Wood (WW) and Vinante-Luyben (VL) models. The PID control optimization is designed for both top product and bottom product. In order to obtain the best optimal values the design process is repeated several times and the mean of the controller values are taken to be $K_{P 1}, K_{I 1}, K_{D 1}, K_{P 2}, K_{I 2}, K_{D 2}$ are recorded. The simulation study is recorded using the MATLAB software to compare with the other algorithms which existed in the literature. In order to have a fair comparison, all the algorithms are assigned with the same population size, iteration number, stopping criteria and objective function.

\section{RESULTS AND DISCUSSTION}

This section presents the performance of FA based PID controller of MIMO for VL model and WW model.The tabulation shows the performance comparison study of BFO, PSO and FA in which the $k_{p}, k_{i}$ and $k_{d}$ values of BFO and PSO are attained from the existing literature. They are tabulated for the values by simulating it for the PID controller for a MIMO process. They are simulated using MATLAB 2013a version.

TABLE 1

The optimal controller values for VL and WW model

\begin{tabular}{|c|c|c|c|c|c|c|c|}
\hline \multirow[t]{2}{*}{ Process } & \multirow[t]{2}{*}{ Method } & \multicolumn{3}{|c|}{ Top product } & \multicolumn{3}{|c|}{ Bottom product } \\
\hline & & $K_{P}$ & $K_{I}$ & $K_{D}$ & $K_{P}$ & $K_{I}$ & $K_{D}$ \\
\hline \multirow{3}{*}{ VL } & FA & -1.1624 & -0.1981 & 0.5807 & 0.8989 & 0.1588 & 0.2182 \\
\hline & $\mathrm{BFO}$ & -1.0174 & -0.4225 & 0 & 2.0935 & 0.4063 & 0 \\
\hline & PSO & -0.8747 & -0.5105 & 0 & 2.1638 & 0.4111 & 0 \\
\hline \multirow{3}{*}{$\mathbf{W W}$} & FA & 12.7069 & 0.4711 & 1.9571 & -10.1500 & -0.4175 & 9.5116 \\
\hline & $\mathrm{BFO}$ & 1.9903 & 0.4028 & 0 & -0.9566 & -0.5088 & 0 \\
\hline & PSO & 2.3552 & 0.3067 & 0 & -1.0345 & -0.4149 & 0 \\
\hline
\end{tabular}

TABLE 2

Comparison with PSO and BFO reference values of VL model

\begin{tabular}{|c|c|c|c|c|c|c|c|c|c|c|c|}
\hline & \multirow{2}{*}{ Method } & \multicolumn{5}{|c|}{ Top product } & \multicolumn{5}{|c|}{ Bottom product } \\
\hline & & ITAE & ITSE & ISE & Mp & Ts & ITAE & ITSE & ISE & Mp & Ts \\
\hline \multirow{3}{*}{ VL } & FA & 5.166 & 2.075 & 1.912 & 0.06 & 16 & 11.24 & 1.661 & 1.388 & 0.07 & 34 \\
\hline & BFO & 19.55 & 4.811 & 2.3 & 0.3 & 28.3 & 5.637 & 0.9216 & 0.9157 & 0.35 & 41 \\
\hline & PSO & 36.38 & 8.537 & 2.786 & 0.45 & 47.3 & 6.563 & 1.067 & 0.9549 & 0.38 & 36 \\
\hline \multirow[b]{3}{*}{ WW } & FA & 1496 & 307.6 & 19.34 & 0.15 & 350 & 619.5 & 193.3 & 17.71 & 0.05 & 210 \\
\hline & BFO & 9644 & 2144 & 42.5 & 0.41 & 450 & 8442 & 1902 & 40.06 & 0.51 & 460 \\
\hline & PSO & 7710 & 1706 & 40.9 & 0.31 & 500 & 6464 & 1455 & 37.52 & 0.41 & 580 \\
\hline
\end{tabular}

The figure 3 (a) and (b), represents the top and bottom product of the VL model using the transfer function existing in the literature. The simulation time is assigned as $100 \mathrm{sec}$ for both top and bottom product of VL distillation model. The performance measure values are tabulated in Table 1.

The figure. 4 (a) and (b), represents the top and bottom product of WW model. The same simulation process is implemented in WW model of distillation column using its transfer function exixting in the literature.Here the simulation time is aasigned as 500sec for both top product and bottom product of WW distillation model. The optimized controller values are tabulated in Table 1 and the corresponding results are presented in Table 2. 


\section{GRAPH}

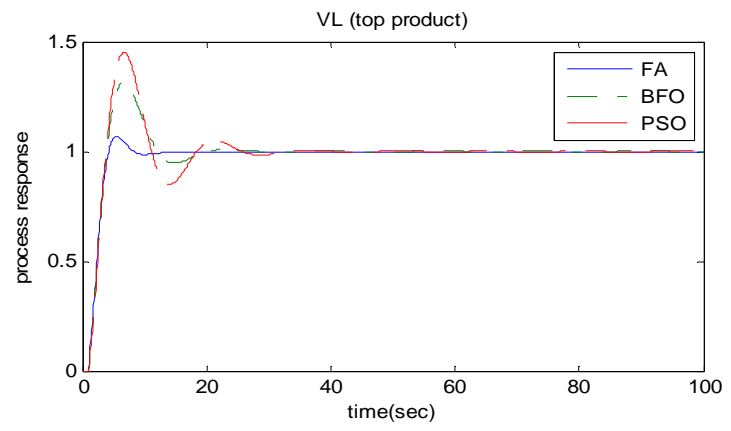

(a) Top product

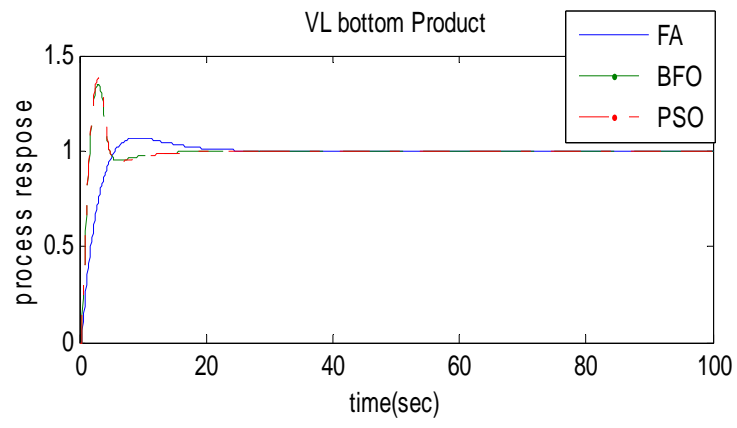

(b) Bottom product

Fig. 3 PID controller response for VL model

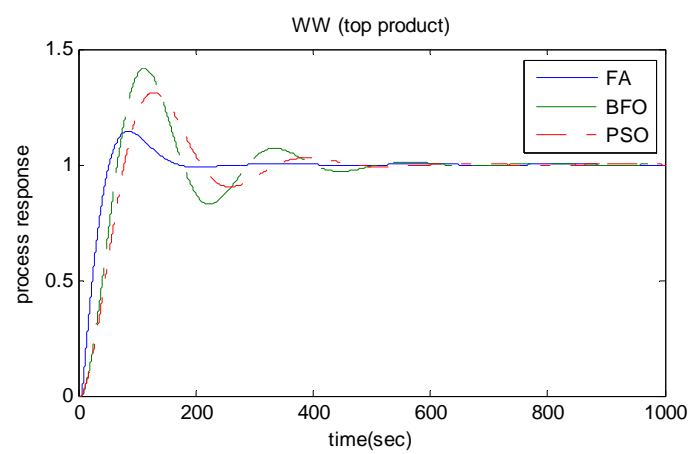

(a) Top product

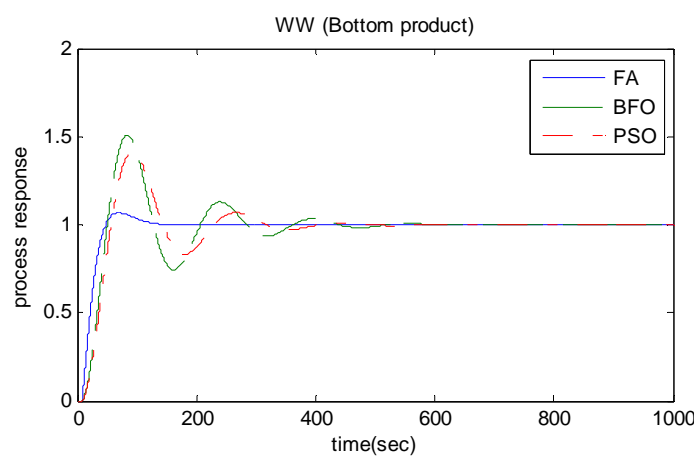

(b)

Bottom product

Fig. 4 PID controller response for WW model

\section{CONCLUSION}

The best possible controller parameters are found for the MIMO system using firefly algorithm are discussed in this paper. The proposed centralised PID controller is tested using the desired benchmark distillation column of WW and VL models. Hence by changing the $\mathrm{Kp}, \mathrm{Ki}, \mathrm{Kd}$ values the response of the system is improved and the graphical representation of the system shows that there is an improvement in the values which gives a lesser peak overshoot and settling time. This study shows that the obtained results provided by the firefly algorithm gives better results when compared with the other existing values existing in the literature.

\section{REFERENCES}

[1] Coelho LS, Mariani VC. Firefly algorithm approach based on chaotic Tinkerbell map applied to multivariable PID controller tuning. Computers and Mathematics with Applications. 2012; 64(8):2371-82.

[2] Latha K, Rajinikanth V. 2DOF PID controller tuning for unstable systems using bacterial foraging algorithm. Lecture Notes in Computer Science. 2012; 7677:519-27.

[3] Manic KS, Devakumar S, Vijayan V, Rajinikanth V. Design of Centralized PI Controller for Interacting Conical Tank System. Indian Journal of Science and Technology. 2016; 9(12): 89920.

[4] Rajinikanth V, Latha K. Controller parameter optimization for nonlinear systems using enhanced bacteria foraging algorithm. Applied Computational Intelligence and Soft Computing. 2012; 2012:12.

[5] Sivakumar R, Balu K. ANFIS based distillation column control. International Journal of Computer Applications Special issue on Evolutionary Computation. 2010; 2:67-73.

[6] Sivakumar R, Suresh MK, Nerthiga V, Akila R, Balu K. Application of fuzzy model predictive control in multivariable control of distillation column. International Journal of Chemical Engineering and Applications. 2010; 1(1):39-42.

[7] Sivakumar R, Rajinikanth V, Sankaran D. Multi-loop PI controller design for TITO system: An analysis with BA, FA, PSO and BFO. Australian Journal of Basic Applications and Sciences. 2015; 9(16):249-54.

[8] Vijayan V, Panda RC. Design of PID controllers in double feedback loops for SISO systems with set-point filters. ISA Transactions. 2012; 51(4):514-21.

[9] Vijayan V, Panda RC. Design of set point filter for minimizing overshoot for low order process. ISA Transactions.2012, 51(2):271-76. 
[10] Rajinikanth V, Latha K. Bacterial foraging optimization algorithm based PID controller tuning for time delayed unstable system. The Mediterranean Journal of Measurement and Control. 2011; 7(1):197-203.

[11] Devikumari AH, Vijayan V. Decentralized PID Controller Design for 3x3 Multivariable System using Heuristic Algorithms. Indian Journal of Science and Technology. 2015; 8(15):1-6.

[12] Rajinikanth V, Satapathy SC. Design of controller for automatic voltage regulator using teaching learning based optimization. Procedia Technology. 2015; 21:295-302.

[13] Latha K, Rajinikanth V, Surekha PM. PSO-Based PID controller design for a class of stable and unstable systems. ISRN Artificial Intelligence. 2013; 2013:11

[14] Karthicraja VM, Sivakumar R, Krishna Prasad VL. PI controller design for TITO process using improved PSO algorithm. International Journal of Applied Engineering Research (IJET). 2015; 10:104-108.

[15] Padma S, Latha K. Multiple-loop PI Controller Design for TITO System using Teaching Learning Based Optimization. Indian Journal of Science and Technology. 2016; 9(12): 89939.

[16] Sivakumar R, Deepa P, Sankaran D. A Study on BFO Algorithm based PID Controller Design for MIMO Process using Various Cost Functions. Indian Journal of Science and Technology. 2016; 9(12): 89942.

[17] Yang XS. Firefly algorithms for multimodal optimization, in Stochastic Algorithms: Foundations and Applications, Lecture Notes in Computer Science. 2009; 5792: 169-178.

[18] Manic KS, Priya RK, Rajinikanth V. Image multithresholding based on kapur/tsallis entropy and firefly algorithm. Indian Journal of Science and Technology. 2016; 9(12): 89949.

[19] Raja NSM, Rajinikanth V, Latha K. Otsu based optimal multilevel image thresholding using firefly algorithm. Modelling and Simulation in Engineering. 2014; 2014:17.

[20] Raja NSM, Manic KS, Rajinikanth V. Firefly algorithm with various randomization parameters: an analysis. Lecture Notes in Computer Science. 2013; 8297:110-121.

[21] Kennedy J, Eberhart R. Particle Swarm Optimization, in proceedings of IEEE International Conference on Neural, 1995: 1942- 1948.

[22] Passino KM.(2002) Biomimicry of bacterial foraging for distributed optimization and control, IEEE Control Systems Magazine, 2002:52-67.

[23] Rajinikanth V, Latha K. Setpoint weighted PID controller tuning for unstable system using heuristic algorithm. Archives of Control Sciences. 2013; 22(4):481-505.

\section{AUTHOR PROFILE}

K.SAROJA is a Student in the Department of Electronics and Instrumentation Engineering St. Joseph's College of Engineering, Chennai - 600119, Tamil Nadu, India. Her area of interest are Process control, industrial instrumentation.

R.STEFKA SHARON is a Student in the Department of Electronics and Instrumentation Engineering, St. Joseph's College of Engineering, Chennai - 600119, Tamil Nadu, India. Her area of interest are Process control, control system.

S MEENA has completed her B.E in National Engineering College, Kovilpatti and her M.E from Sathyabama University with specialistion in Electronics \& Control. She has 11 years of experience in teaching. Her areas of research are Process control and embedded systems.

K CHITRA: Currently Dr. K. Chitra is working as a Professor, in the SENSE at VIT, Chennai. Dr. K. Chitra received her B.E. Degree in Electronics and communication Engineering from Bharathiar University, Coimbatore in 1990, M.E. Degree in Applied Electronics from Bharathiar University in 1992 and Ph.D. degree in Optical Communication from Anna University Chennai in 2008. Dr. K.Chitra has spent her 26 years of experience in teaching and guiding projects for undergraduate and postgraduate students. Dr. K. Chitra has added 15 international publications to her credit. She has few funded projects from Government of India. Dr. K. Chitra's areas of interests include optical communication, optical networks, wireless sensor and computer networks, Biomedical Engineering and microwave Engineering. 